\title{
Keratoconus und dessen Behandlung mit Hornhautvernetzung und Kontaktlinsen: ein Überblick
}

\author{
U. Scheschy $\cdot$ H. Scheschy
}

Eingegangen: 22. November 2015 / Angenommen: 26. Dezember 2015 / Online publiziert: 28. Januar 2016

(c) Die Autor(en) 2016. Dieser Artikel ist auf Springerlink.com mit Open Access verfügbar.

Zusammenfassung Keratoconus (KC) ist die häufigste primäre Ektasie der Hornhaut $(\mathrm{HH})$. Die Erkrankung beginnt in der Regel zwischen dem 10. und 20. Lebensjahr, jedoch wurde auch früheres und späteres Auftreten beschrieben; er befällt beide Geschlechter und alle Ethnien. Das geschätzte Vorkommen beträgt 1:2000. Keratoconus-Befunde und -Symptome sind abhängig von der Erkrankungsschwere. Frühe Stadien können ohne sorgfältige Diagnostik unbemerkt bleiben. Die Erkrankungsprogression ist assoziiert mit Zunahme der Myopie und des Astigmatismus und Verlust an Sehschärfe infolge Irregularität der Hornhautoberfläche, welche mit Brille nicht kompensiert werden kann. Der Sehverlust entsteht hauptsächlich durch den irregulären Astigmatismus und erst sekundär infolge Hornhauttrübungen und -narben. Die zentrale Hornhaut- und Epithelverdünnung geht häufig der zentralen Ektasie voraus. In fortgeschrittenen Fällen ist oft eine bogenoder ringförmige Linie um die Conus-Basis zu sehen (Fleischer-Ring), weitere Zeichen sind Vogt-Streifen, diese sind feine zentrale, vertikale Streifen in der Descemet-Membran.

Ein wesentliches Kennzeichen des Keratoconus ist die Ausdünnung (verminderte Dicke) der zentralen Anteile der Hornhaut, die eine geringere biomechanische Stabilität verursacht. Durch diese kommt es in den verdünnten Bereichen durch den Augeninnendruck zur stärkeren Vorwölbung (Protrusion), was eine kegelförmige Kontur der betroffenen Hornhaut bewirkt. Die genauen Ursachen des Keratoconus sind derzeit noch nicht sicher bekannt. Neben der genetischen Prädisposition scheinen

U. Scheschy $(\bowtie) \cdot$ H. Scheschy

Wien, Österreich

E-Mail: ulrike.scheschy@meduniwien.ac.at

H. Scheschy

E-Mail: herbert.scheschy@aon.at zusätzliche biomechanische und biochemische Faktoren für den Ausbruch der Erkrankung verantwortlich zu sein.

Natürlich wäre es vielversprechend, das Fortschreiten des KC durch eine corneale crosslinking-Therapie zu verhindern. Die möglichen Komplikationen dieser Therapie werden besprochen. Solange mit Brillenkorrektur eine für den Patienten ausreichende Sehschärfe erreicht werden kann, sollte der Sehfehler mit Brille korrigiert werden; erst wenn das nicht mehr genügt, halten wir die Anpassung rigider Kontaktlinsen für indiziert.

Der Artikel soll einen Überblick über die klinischen Kennzeichen, subjektive Auswirkungen, Diagnostik, Verlauf, Pathogenese und Therapie-Management mit Hornhautvernetzung und Kontaktlinsen geben.

Schlüsselwörter Keratoconus · Diagnostik • Verlauf . Pathogenese · Vernetzung $\cdot$ Kontaktlinsen

\section{Keratoconus and its treatment with corneal crosslinking and contact lenses: A review}

Summary Keratoconus is the most common primary ectasia of the cornea. Generally the disease starts between the ages of 10 and 20, however, earlier and later occurrences were also reported; it affects both genders and all ethnic groups. The estimated prevalence in the general population is 1 per 2000. Keratoconus findings and symptoms are depending on disease severity. Early stages can remain unnoticed without careful diagnostic. Disease progression is associated with increased myopia and astigmatism and loss of visual acuity due to surface irregularity, which cannot be compensated with spectacles. Vision loss is primarily caused by irregular astigmatism and secondarily due to corneal haze and scars. Central corneal stroma and epithelial thinning frequently precedes ectasia. In advanced cases an arc or circle line (Fleischer's ring) can often be seen around the cone base. Further signs are Vogt's striae, 
which are fine vertical lines at the Descemet membrane. A main characteristic of keratoconus is the thinning (reduced thickness) of the central parts of the cornea causing a diminished bio-mechanical stability. This leads to a greater protrusion in the thinned out areas via the internal eye pressure causing a conical contour of the affected cornea. The exact causes of keratoconus are presently not known with certainty. It seems that apart from the genetic predisposition additional bio-mechanical and biochemical factors are responsible for the occurrence of the disease.

Of course it would be tempting to prevent the progression of keratoconus by corneal crosslinking therapy - the possible side effects of this therapy are discussed. As long as adequate visual acuity for the patient can be achieved by means of corrective spectacles, the visual defect should be corrected with spectacles; only in cases where this is not sufficient we consider the fitting of rigid contact lenses indicated. This paper should provide an overview on the clinical characteristics, subjective effects, diagnosis, progression, pathogenesis and therapy management with contact lenses.

Keywords Diagnosis · Progression · Pathogenesis · Contact lenses · Corneal crosslinking

\section{Definition}

Der Keratoconus ist eine degenerative Erkrankung der Hornhaut $(\mathrm{HH})$, welche mit einer zentralen oder parazentralen Hornhautverdünnung, einer apikalen Protrusion der Hornhaut und einem irregulären Astigmatismus einhergeht und $\mathrm{zu}$ einer Sehverschlechterung führt [1]. Eine adäquate Erstbeschreibung des KC bei deutlicher Unterscheidung von anderen ektatischen HH-Erkrankungen erfolgte durch Nottingham 1854 [2].

Die wichtigsten Eigenschaften der normalen Hornhaut sind:

1. Transparenz: damit das Licht ungehindert passieren kann.

2. Kurvatur: sie ermöglicht die erforderliche Brechkraft, zusammen mit der Augenlinse, damit das Bild der Außenwelt möglichst scharf auf der Netzhaut abgebildet wird.

3. Rigidität: sie bietet gemeinsam mit den Lidern einen gewissen Schutz gegen Perforation und dient der Stabilisierung der Brechkraft.

Bei Prädisposition und zusätzlichen äußeren Einflüssen kann es zur Änderung dieser drei Eigenschaften der HH kommen in Form einer Erkrankung, die als KC bezeichnet wird. Dabei ist zu betonen, dass bei jungen Menschen bis etwa um das 20. Lebensjahr die Rigidität der $\mathrm{HH}$ noch gering ausgeprägt ist, weil in diesem Alter nur eine geringe Quervernetzung der HH-Fibrillen besteht, sodass eine erhöhte Anfälligkeit für Formveränderungen der Kurvatur vorhanden ist.
KC ist die häufigste primäre Ektasie meist beider Augen, jedoch mit asymmetrischer Ausprägung [3, 4]. Die Hornhautdegeneration ist charakterisiert durch zentral lokalisierte Hornhautverdünnung, welche zur Protrusion im verdünnten Bereich führt. Die Hornhautverdünnung vollzieht sich normalerweise infero-temporal oder auch in der zentralen Kornea [5, 6]. Die Protrusion verursacht eine Progression der Myopie und einen irregulären Astigmatismus, der die Sehqualität beeinträchtigt $[7,8]$. KC wird meist während der Pubertät erkennbar, es sind aber auch Fälle von früherem und späterem Beginn bekannt $[7,8]$ Nur in einem Teil der Fälle [6] schreiten die typischen $\mathrm{HH}$-Veränderungen fort bis etwa um das 35.-40. Lebensjahr, ab dem die Erkrankung gewöhnlich stabilisiert [7]. Bei unilateralem KC kann sich auch am sog. guten Auge (Auge mit forme fruste KC) in einem Teil der Fälle ein progredienter KC entwickeln [9], es kann jedoch auch stabil bleiben [10].

\section{Klinische Kennzeichen}

Die wichtigsten klinischen Kennzeichen des KC sind die zentrale oder parazentrale Hornhaut-Verdünnung, die apikale Protrusion der Hornhaut und der irreguläre Hornhaut-Astigmatismus.

Die Augensymptome und Zeichen des KC variieren abhängig von der Schwere der Erkrankung. Bei KC zeigen sich im Anfangsstadium normalerweise keine Symptome, sodass die Erkrankung für Patient und Augenarzt unbemerkt bleiben kann ohne spezifische TestUntersuchungen [9, 11, 12]. Die Erkrankungsprogression manifestiert sich in Form eines signifikanten Verlustes an Sehschärfe, welcher mit Brille nicht mehr gebessert werden kann. Da es viele Ursachen von verminderter Sehschärfe gibt, sollte der Augenarzt auch an KC denken und die notwendigen Untersuchungen zur KC-Diagnostik durchführen. Der Verlauf des KC ist individuell äußerst variabel und unterschiedlich und kann von kaum fortschreitender Entwicklung bis zu sehr rascher Progredienz bis zur Notwendigkeit einer Operation reichen. In moderaten und fortgeschrittenen Fällen von KC wird häufig ein bogenförmiger oder zirkulärer HämosiderinGrenzstreifen um die Conus-Basis (Fleischer Ring) [13] sichtbar. Ein weiteres charakteristisches Zeichen ist das Auftreten von Vogt-Streifen, die als feine vertikale Linien im Hornhautmikroskop erkennbar sind [14]. Häufige Zeichen sind auch oberflächliche und tiefe Hornhauttrübungen und Narben bei fortgeschrittenen Stadien der Erkrankung [15]. Signifikante Hornhauttrübungen und Narben können bei KC zusätzlich auch durch LangzeitTragen von CL verursacht werden.

\section{Subjektive Auswirkungen des KC für den Betroffenen}

Die irreguläre Hornhaut-Verkrümmung führt $\mathrm{zu}$ verzerrtem Sehen, zu Doppel- oder Mehrfachbildern und 
damit zur Verschlechterung des Sehvermögens, insbesondere in der Dämmerung und Dunkelheit. In fortgeschrittenen Stadien der Erkrankung kommt es zu feinen Rissen der Bowman-Membran, deren Heilungsprozesse zu Narben und Trübungen im Bereich des Konus-Apex führen. In der Regel entwickelt sich der KC im Pubertätsalter bis zum jungen Erwachsenenalter, aber auch früheres oder späteres erstmaliges Auftreten ist möglich. Er schreitet dann über Jahre mehr oder weniger stark fort und tendiert dann um das 40 . Lebensjahr zum Stillstand. Bei einem erheblichen Teil der frühen KC-Fälle kommt es jedoch nicht zur Progression der Erkrankung und deren leichte KC-Hornhaut-Veränderungen bleiben dauerhaft stabil.

\section{Diagnostik}

Die Diagnose KC wird durch Messung der Oberflächenkrümmung (Topographie) und des Dickenprofils (Pachymetrie) der Hornhaut gestellt. Bei KC zeigt die Topographie ein typisches Wölbungsmuster und bei der Pachymetrie findet sich am Apex des KC eine Verdünnung der Hornhaut [16-18].

Oft wird ein KC zufällig beim Augenarztbesuch entdeckt. Viele Patienten mit KC gehen aber auch zum Augenarzt, weil sie vermuten, dass ihre Brillenstärke nicht mehr stimme und sie eine langsam zunehmende Verminderung der Sehschärfe bemerken. Meist tritt eine Zunahme der Myopie und des Astigmatismus auf, bei weiterer Progression kommt es zur Irregularität der Hornhaut-Oberfläche. Wenn die Hornhautverformung zunimmt, kann mit Brillenkorrektur keine zufriedenstellende Sehschärfe mehr erzielt werden. Da es viele Ursachen für Visusminderungen gibt, ist es wichtig, dass der behandelnde Augenarzt an KC als mögliche Ursache denkt und die KC-Diagnostik durchführt. Bei irregulärem Astigmatismus können nur mehr rigide Kontaktlinsen (CL) das Sehen verbessern.

Die CL gleicht die unregelmäßige Oberfläche der Hornhaut mit Hilfe der zwischen Linsenhinterfläche und Hornhautvorderfläche befindlichen Tränenflüssigkeit aus, sodass das optische Gesamtsystem, bestehend aus CL, Hornhaut, Kammerwasser, Pupille, Augenlinse und Glaskörper, wieder ein nahezu scharfes Bild auf der Fovea centralis der Netzhaut entstehen lässt. Durch die reguläre optische Oberfläche der CL wird der irreguläre Astigmatismus der Hornhaut egalisiert.

Differentialdiagnostisch sollte vor Einleitung therapeutischer Maßnahmen bei KC (z. B. collagen crosslinking) ein Pseudokeratoconus (corneal warpage) bei CL-Trägern durch eine mehrwöchige CL-Karenz ausgeschlossen werden $[11,19,20]$.

Bei der Untersuchung mit der Spaltlampe sind im Frühstadium des KC keine Hornhautveränderungen $\mathrm{zu}$ erkennen. Mit Keratometrie unter Verwendung eines manuellen Keratometers (z. B. Javal-Schiötz) zeigen sich eine steile Hornhaut-Kurvatur (kurze Krümmungsradien) und/oder verzerrte Miren bei KC.
Dieses Gerät kann als einfache Bildgebungsmethode verwendet werden. Erst bei der Untersuchung mit der Videokeratographie (=Placido-Topographie) gelang es in der Mitte der 1980er Jahre erstmals ein Profil der Hornhautvorderfläche zu erstellen, ähnlich wie mit den Keratometern, die auch Reflexbilder des Tränenfilms vermessen lassen. Das funktioniert aber nur bei intaktem Tränenfilm, welcher bei KC oft nicht gegeben ist. Auch können periphere Hornhautbereiche mit dem Videokeratoskop nicht mehr vermessen werden, da der Meßbereich auf $9 \mathrm{~mm}$ beschränkt ist [21].

2003 präsentierte die Firma Oculus, Wetzlar, erstmals die Scheimpflugkamera „Pentacam“, [22] mit deren Hilfe eine ganzflächige Pachymetrie, eine Hornhauttopographie von Hornhautvorder- und Rückfläche, eine dreidimensionale Vorderkammeranalyse, sowie eine Katarakt-Analyse durchgeführt werden kann. Diese Kamera eignet sich zur Keratoconusdiagnostik und als bildgebendes Verfahren des vorderen Augenabschnitts. Ebenso bietet das Orbscan-System, Bausch und Lomb [19] in Verbindung mit der Spaltlampe eine Hornhauttopographie und Ultraschallpachymetrie an. Durch Vergleich der verschiedenen Messergebnisse mit Normdaten lässt sich die Diagnose KC erarbeiten und es können auch Frühformen des KC, sog. „forme fruste“ sicher erkannt werden [22, 23].

Aber auch die Videokeratographie, die optische Kohärenztomographie des Augenvordersegments $[21,24]$ und die Ultraschallpachymetrie leisten wertvolle Beiträge zur Diagnostik. Die Überprüfung der KCStabilisierung oder Progression erfordert sowohl die vergleichende topographische Messung der Hornhautkurvatur als auch der Hornhautdicke. Voraussichtlich werden in der Zukunft mit den neuen diagnostischen Möglichkeiten deutlich mehr „forme fruste“ Fallzahlen festgestellt werden als bisher in der Statistik aufschienen.

Weil sich das biomechanische Verhalten der Hornhaut bei KC stark verändert, wurden in den letzten Jahren Apparate entwickelt, die es ermöglichen, dieindividuellen biomechanischen Eigenschaften der Hornhaut in vivo zu analysieren. Besonders die Früherkennung des subklinischen KC erfordert neben der Topographie und Pachymetrie die zusätzliche Berücksichtigung der Biomechanik der Hornhaut. Mit dem Ocular-ResponseAnalyser (Ora Reichert Ophthalmic Instruments, Buffalo, USA) lassen sich die biomechanischen Parameter der Hornhaut in vivo ermitteln [25].

Mit einem weiteren Gerät, dem Corvis ST (Fa. Oculus Wetzlar) lassen sich der Augeninnendruck, die Hornhautdicke und auch die individuellen biomechanischen Eigenschaften der Hornhaut analysieren.

\section{Verlauf}

Die Erkrankung verläuft häufig langsam zunehmend. Beginnt die Hornhautverdünnung schon vor dem 18. Lebensjahr, so muss man mit einer rascheren Zunahme der Hornhaut-Veränderungen rechnen. Generell kann 
etwa bei $75 \%$ der Betroffenen mit CL ein ausreichendes Sehvermögen erhalten werden [9]. Bei weiterer Zunahme der kegeligen Hornhautverformung kommt es meist zu narbigen Trübungen des zentralen Hornhautbereiches, sodass auch mit CL die Sehschärfe nicht mehr ausreichend gebessert werden kann. Dann ist der Zeitpunkt für eine Hornhauttransplantation gekommen.

Die Indikation zur Hornhauttransplantation und der Operationszeitpunkt müssen für jeden Patienten individuell überlegt und mit der jeweiligen Lebenssituation des Patienten übereingestimmt werden. Das Ziel der Behandlung sollte es sein, die eigene Hornhaut des Patienten solange wie möglich zu erhalten.

\section{Zur Pathogenese des KC}

Die Ursachen für die Entwicklung des KC sind derzeit noch nicht sicher bekannt. Die Forschung zeigt eine Reihe von Ansätzen, die auf auslösende Faktoren hindeuten. Es gibt viele Hinweise für eine genetische Ursache des KC. Etwa $13 \%$ der KC-Patienten haben mindestens ein weiteres betroffenes Familienmitglied [26]. Mehrere Gene konnten bisher identifiziert werden, die mit KC in Verbindung stehen. Auch gibt es mehrere Krankheiten, bei denen überdurchschnittlich häufig auch ein KC vorkommt [27-29]. Dazu gehören die atopische Dermatose, Trisomie 21 (Down-Syndrom), Monosomie X (Turner Syndrom), Lebersche kongenitale Amaurose, Kollagenosen, Retinopathia pigmentosa, Marfan Syndrom und Mitralklappenprolaps [30-33]. Das Phänomen der CL-induzierten Stromaverdünnung mag abhängig sein vom Linsen-induzierten KeratozytenVerlust, im Hinblick auf die Rolle der Keratozyten für die Aufrechterhaltung der strukturellen Integrität der Hornhaut. Der gegenwärtige Konsens besagt, dass Langzeittragen von CL zur Keratozyten-Apoptose führt, infolge von chronischem Sauerstoffmangel und chronischen Mikrotraumata der Hornhaut [34].

$\mathrm{Ku}$ JY, et al. [35] aus Neuseeland haben berichtet, dass die Keratozytendichte bei $\mathrm{KC}$ signifikant niedriger ist als bei Patienten mit normaler Hornhaut. Bei KC-Patienten, die längere Zeit rigide KL trugen, war die Keratozytendichte signifikant noch niedriger als bei denen, die keine KL trugen $[35,36]$. Die niedrigere Keratozytendichte zeigt sich in Form einer Verdünnung des Hornhautstromas, vor allem im Apexbereich. Zusätzlich zeigt sich eine Epithelverdünnung im Apexbereich [37-40], die durch das Tragen von rigiden CL noch verstärkt wird. Bei fortschreitender Erkrankung kommt es zu irregulär konfigurierten und kernhaltigen Epithelzellen [41].

Die Unterscheidung zwischen der durch die KC-Erkrankung und CL-induzierter Hornhautverdünnung haben Pflugfelder et al. [42] untersucht. Es wurde nachgewiesen [36], dass bei KC eine erhöhte KeratozytenApoptose stattfindet, die durch das Tragen rigider CL noch verstärkt und dadurch die Dicke und Stabilität des Hornhautgewölbes geschwächt wird. Keratozyten liefern ja die Grundsubstanz des Kollagens, aus dem die kollagenen Fibrillen und die Interzellularsubstanz des Hornhautstromas synthetisiert werden [36, 42-44]. Macsai et al. [45] berichteten über die Induktion eines „echten“ KC bei Patienten durch Langzeittragen von KL.

Diese Überlegungen aus der Mikrobiologie, die durchaus logisch scheinen, könnten Anlass dafür sein, bei KC die Refraktionsanomalie solange wie möglich mit Brille zu korrigieren. Alle möglichen Ursachen der Erkrankungsprogression sollten eruiert werden.

Das veränderte und verdünnte Hornhautepithel scheint für die Pathogenese des KC eine wichtige Rolle zu spielen, weil es seine Schutzfunktion für die Keratozyten gegen schädliche Umwelteinflüsse nicht mehr voll erfüllen kann. Es gibt Grund zur Vermutung, dass die verminderte Keratozytendichte und die langsam zunehmende Stromaverdünnung eine sekundäre Folge des verdünnten und morphologisch veränderten Epithels sein könnten.

\section{Pathologie}

Das Epithel zeigt eine zentrale Verdünnung mitirregulärer oder verdickter Basalmembran und Defekte in der Bowman-Schicht. Stromale Narben und Zellen wurden in der Nähe dieser Defekte festgestellt und KeratozytenApoptose wurde nachgewiesen [46-48]. Die in-vivo confocale Mikroskopie hat verminderte subbasale Nervendichte gezeigt, die mit verminderter Hornhautsensibilität und verminderter Basal-Epithelzelldichte einhergeht [49]. Es besteht ein Verlust von stromalen Kollagen-Lamellen und eine gestörte Ausrichtung der Kollagenfibrillen. Verminderte Keratozytendichte zeigt sich insbesondere im zentralen vorderen Stroma [50].

\section{Therapie-Management}

Eine Heilung des KC ist gegenwärtig nicht möglich. Seine Folgen für das Sehvermögen lassen sich mit unterschiedlichen Hilfsmitteln verbessern. Es gibt Methoden zur Verbesserung des Sehvermögens und andere, die den KC ursächlich behandeln. Solange man ohne optische Korrektur oder mit einer Brille oder weicher Kontaktlinse eine Sehschärfe erreichen kann, die den Anforderungen des Patienten genügt, erscheint das als gute Lösung. Wenn sich aber ein irregulärer Astigmatismus entwickelt hat, lässt sich mit Brillenkorrektur oder weicher CL kein ausreichendes Sehvermögen mehr erzielen. In dieser Situation ist das Tragen rigider CL erforderlich, es sei denn die Progression des KC wurde vorher mit der neu entwickelten corneal cross-linking (CXL) Therapie gestoppt.

\section{Quervernetzung (Corneale cross-linking Therapie)}

Das Verständnis der Pathologie und Pathogenese des KC hat sich weiter entwickelt. Spoerl et al. [51] haben als 
erste den Effekt einer Kombination von Riboflavin als Photosensitizer und UV-A-Licht im Labor-Experiment zur cornealen Kollagen-Quervernetzung verwendet. Eine Verstärkung der Hornhautrigidität wurde berichtet, sowohl bei Schweine- als auch bei menschlichen Hornhäuten [51-53].

In der Folge wurden Ergebnisse der ersten klinischen Pilot-Prüfung von Wollensak et al. (Dresden) [54] veröffentlicht. Bei einem follow-up von 3-47 Monaten wurde die Progression des KC in allen 23 behandelten Augen gestoppt. Eine mittlere Reduktion von 2,01 Dioptrien der maximalen Keratometrie und 1,14 Dioptrien des Refraktionsfehlers wurde beobachtet in den 16 Augen, die eine Regression zeigten. Zweck der Studie war zu eruieren, ob CXL geeignet ist, den natürlichen Verlauf des KC zu verändern. Komplikationen der CXL-Therapie inkludieren verzögerte Wundheilung, bakterielle oder Pilzinfektionen, Akanthamoeben und sterile Keratitis [55-61], bullöse Keratopathie und Herpes Reaktivierung. Bei dünnen Hornhäuten unter $400 \mu \mathrm{m}$ kann nach CXLBehandlung ein signifikanter Endothelzell-Verlust entstehen [62]. Zusätzliche Komplikationen wurden berichtet in Form von persistierendem Hornhautödem und Hornhaut-Dekompensation mit Zeichen von Schäden der Strukturen des vorderen Augensegments nach einer CXL-Therapie [63-66].

Es erscheint durchaus verständlich, dass es nach einer CXL-Therapie zu Komplikationen kommen kann, weil die Transparenz und die Gesamtfunktion der Hornhaut nur durch das störungsfreie Zusammenwirken aller Gewebsschichten gewährleistet ist. Eine Schädigung der Epithelstammzellen am Hornhautlimbus durch die CXLBehandlung scheint möglich zu sein.

Vazirani et al. [67] fassen den gegenwärtigen Stand des Wissens über die CXL-Therapie folgendermaßen zusammen:

Zusammenfassend ist Collagen-crosslinking eine Möglichkeit zum Aufhalten der KC-Progression und zweifellos gesichert durch Labor- und klinische Studien. Weitere gutgeplante prospektive, randomisierte klinische Untersuchungen mit genauen Fall-Definitionen, geeigneten Ergebnismessungen und ausreichendem follow-up wären erstrebenswert. Zur Zeit laufen multiple klinische Prüfungen der Phase II und III mit bislang vielversprechendem Verlauf hinsichtlich der Wirksamkeit der CXL-Therapie.

Gegenwärtig besteht kein Konsens darüber, was Progression bei KC begründet. Das eröffnet ein Potential für Missbrauch in Form ungerechtfertigter Prozeduren. Sachdienliche Daten sind nicht verfügbar, die zeigen, dass Collagen-crosslinking die Anzahl notwendiger Keratoplastiken reduziert, die CL-Toleranz verbessert oder aufrecht erhält. Unser Verständnis für Keratozytenverlust in der Hornhaut legt nahe, dass der Effekt von Crosslinking vorübergehend sein mag. In Hinblick auf berichtete Nebenwirkungen empfehlen wir striktes Festhalten an Pachymetrie-Richtwerten und Crosslinking Protokollen. Der erwartete Nutzen und potentielle Risiken sollten klar mit dem Patienten diskutiert werden, um ihn in die Lage zu versetzen, gut informiert seine Auswahl zu treffen.
Unseres Wissens gibt es derzeit noch keine in vivoStudien, welche die Auswirkungen der CXL-Therapie auf die menschlichen limbalen Epithel-Stammzellen evaluierten. Der Schutz der limbalen Stammzellen während der CXL-Prozedur sollte in Erwägung gezogen werden, insbesondere deshalb, weil bei KC a priori die zentrale Epithelzelldichte vermindert ist $[46,68]$.

\section{Optische Korrektion}

In frühen Stadien des KC kann die Refraktionsanomalie mit Brille korrigiert werden. Wenn jedoch die Erkrankung fortschreitet (was nicht immer der Fall ist), kommt es zur Veränderung der Hornhaut-Form, woraus sich ein irregulärer Astigmatismus ergibt, der mit Brille nur mehr unzureichend korrigiert werden kann. Deshalb ist in dieser Situation die Verwendung rigider Kontaktlinsen notwendig. Diese sind die meist verwendete CL-Type bei KC. Zwei große Studien $[9,11]$ zeigten die häufige Verwendung rigider gaspermeabler CL. Eine Reihe von Linsen-Designs sind verfügbar und bringen einen zufriedenstellenden Tragekomfort. Rigide CL gleichen die Irregularität der Hornhaut-Kurvatur aus, verbessern die Sehschärfe erheblich und sorgen bei adäquater Anpassung auch für einen guten Austausch der Tränenflüssigkeit zwischen Linse und Hornhaut-Oberfläche. Ein beliebtes Design ist die RoseKLinse (Menicon Co, Ltd, Nagoya, Japan).

Es wurde allerdings berichtet [69], dass CL die Hornhaut-Physiologie auf mehrfache Weise beeinträchtigen. Durch Langzeittragen rigider gaspermeabler CL wird die Hornhaut dünner und es kommt zur Versteilung der Kurvatur (Verkürzung der zentralen Krümmungsradien). Zusätzlich kommt es zur Irregularität der Hornhaut-Oberfläche.

In schweren Fällen von KC, wenn sich zentrale hornhautnarben und -trübungen entwickelt haben, kann auch mit rigiden CL das Sehvermögen nicht mehr gebessert werden. In solchen Fällen kann der betroffene Patient nur mehr mit Hilfe einer lamellierenden oder perforierenden Keratoplastik durch den erfahrenen Augenchirurgen wieder gutes Sehvermögen erlangen.

\section{Komplikationen des Kontaktlinsen-Tragens}

Komplikationen des CL-Tragens können als Folge von mechanischer Schädigung, Hypoxie oder hypokapnischem Stress, immunologischen Reaktionen auf Linsenablagerungen oder Linsenlösungen, toxischen Reaktionen auf Lösungen, oder Infektionen entstehen und können exazerbieren durch lokale Augenprobleme (z. B. trockene Augen) oder systemische Funktionsstörungen (z. B. Diabetes). Das Phänomen der CL-induzierten stromalen Verdünnung mag abhängig sein vom Linsen-induzierten Keratozytenverlust in Hinblick auf die Rolle der Keratozyten zur Aufrechterhaltung der Struktur-Integrität der Hornhaut. 
Der gegenwärtige Konsens ist folgender, dass CLTragen zu Keratozyten-Apoptose führt. Mechanische Stimulation der Hornhautoberfläche durch die physikalische Präsenz einer CL und das konsequente Freisetzen von inflammatorischen Mediatoren sind wahrscheinlich die Hauptgründe für die beobachtete Reduktion der Anzahl der Keratozyten [34].

Unter der Berücksichtigung der Publikation von Efron über die Untersuchung der Hornhaut mittels der confocalen- Mikroskopie bei Langzeitträgern von CL und aufgrund eigener Erfahrungen [10] sind wir der Meinung, dass bei KC bei jungen Menschen bis zum 20. Lebensjahr noch keine CL getragen werden sollten, weil die Hornhaut im jugendlichen Alter noch zu wenig Rigidität entwickelt hat und die bei $\mathrm{KC}$ primär schon verdünnte $\mathrm{HH}$ durch CL leichter irregulär verformt werden kann.

Bei Patienten mit forme fruste KC sollte der Sehfehler so lange wie möglich mit Brille korrigiert werden, um die Entwicklung einer Irregularität der $\mathrm{HH}$ durch $\mathrm{CL}$ nicht zu fördern. Aus dem gleichen Grund sollte bei unilateralem KC das kaum vom KC betroffene (forme fruste) Auge möglichst lange oder dauernd mit Brille korrigiert werden [10].

Es darf nicht durch Frühanpassung rigider CL bei Frühformen des KC die langfristige Prognose der Erkrankung verschlechtert werden, weil der KC in einem erheblichen Teil der Fälle dauerhaft im Frühstadium stationär bleibt [6]. Hingegen begünstigt das Langzeittragen rigider CL die Entwicklung zentraler Hornhauttrübungen, die dann eine Keratoplastik erforderlich machen.

\section{Konklusion}

In Hinblick auf das CL-Tragen bei KC sind wir zur Überzeugung gekommen, dass bei Vorhandensein eines irregulären Astigmatismus rigide CL ein unentbehrliches Hilfsmittel zur Verbesserung des Sehvermögens sind. Wenn jedoch mit Brillenkorrektur noch ein den Bedürfnissen des Patienten genügendes Sehvermögen erreicht werden kann, halten wir es für angebracht, die Brillenkorrektur solange wie möglich beizubehalten, um die Nebenwirkungen und möglichen Komplikationen des Langzeit-Tragens von CL zu minimieren. Die Zukunft wird zeigen, ob die corneale crosslinking-Therapie oder die rechtzeitig durchgeführte tiefe anteriore lamelläre Keratoplastik die auf Dauer erfolgreichere Behandlungsmethode des progressiven KC darstellt.

\section{Open Access}

Dieser Artikel unterliegt den Bedingungen der Creative Commons Attribution License. Dadurch sind die Nutzung, Verteilung und Reproduktion erlaubt, sofern der/die Originalautor/en und die Quelle angegeben sind.

\section{Einhaltung ethischer Richtlinien}

\section{Interessenkonflikt}

Die Autoren U. Scheschy, H. Scheschy erklären, dass kein Interessenkonflikt bezüglich der Themen, welche in diesem Artikel diskutiert werden, besteht.

\section{Literatur}

1. Theuring A, Raiskup F, Spörl E, et al. (2013) 10-Jahresergebnisse nach Hornhautvernetzung mit Riboflavin/UVA bei Patienten mit Keratoconus. Jahrestagung der Sächsischen Augenärztlichen Gesellschaft 2013.

2. Nottingham J. Practical observations on conical cornea and on the short sight, and other defects of vision connected with it. London: J Churchill; 1854.

3. Chopra I, Jain AK. Between eye asymmetry in keratoconus in an Indian population. Clin Exp Optom. 2005;88(3):146-52.

4. Auffarth GU, Wang L, Völcker HE. Keratoconus evaluation using Orbscan topography system. J Cataract Refract Surg. 2000;26(2):222-8.

5. Prisant O, Legeais RG. Superior keratoconus. Cornea. 1997;16(6):693-4.

6. Choi JA, Kim MS. Progression of keratoconus by longitudinal assessment with corneal topography. Invest Opthalmol Vis Sci. 2012;53(2):927-35.

7. Rabinowitz YS. Keratoconus. Surv Ophthalmol. 1998;42(4):297-319.

8. Kennedy RH, Bourne WM, Dyer JA. A 48-year clinical and epidemiologic study of keratoconus. Am J Ophthalmol. 1986;101(3):267-73.

9. Wagner H, Barr Jt, Zadnik K. Collaborative longitudinal evaluation of keratoconus (CLEK) study: methods and findings to date. Cont Lens Anterior Eye. 2007;30(4):223-32.

10. Scheschy U, Scheschy H. Langzeit-Verlaufsbeobachtung des Keratoconus bei Unilateralität. Spektrum Augenheilkd. 2014;29(3):109-17.

11. Weed KH, MacEwen CJ, Giles T, et al. The Dundee university Scottish keratoconus study demographics, corneal signs, associated diseases, and eye rubbing. Eye. 2008(2);22:534-41.

12. Owens H, Gamble GD, Bjornholdt MC, et al. Topogrphic indications of emerging keratoconus in teeagers New Zealanders. Cornea. 2007;26(3):312-8.

13. Fleischer B. Über Keratoconus und eigenartige Pigmentbildung in der Kornea. Münchner Med Wschr. 1906;53:625-26.

14. Hollingworth JG, Efron N. Observation of banding pattern (Vogt Striae) in keratoconus: a confocal microscopy study. Cornea. 2005;24(2):162-6.

15. Zadnik K, Barr JT, Gordon MO, et al. CLEK Study group. Biomicroscopic signs and disease severity in keratoconus. Cornea. 1996;15(2):139-46.

16. Li Y, Meisler DM, Tang M, et al. Keratoconus diagnosis with optical coherence tomography pachymetry mapping. Ophthalmology. 2008;115(12):2159-66.

17. Gobbe $M$, Guillon $M$. Corneal wavefront aberration measurement to detect keratoconus patients. Cont Lens Anterior Eye. 2005;28(2):57-66.

18. Emre S, Doganay S, Yologlu S. Evaluation of anterior segment parameters in keratoconic eyes measured with Pentacam System. J Cataract Refract Surg. 2007;33(10):1708-12.

19. Hartstein J. Corneal warping due to wearing of corneal contact lenses. A report of 12 cases. Am J Ophthalmol. 1965;60(6):1103-4. 
20. Spaniol K, Roth M, Stupp T. Acute alteration of corneal topography. Ophthalmologe. 2014;111(7):672-75.

21. Belin MW, Khachikian SS. An introduction to understanding elevation- based topography:how elevation data are displayed-a review. Clin Exp Ophthalmology. 2009;37(1):14-29.

22. ReisdorfS. Scheimpflugkamera-Principle and Applications. ZPA. 2011;12:557-65.

23. Bühren J. Corneal topography and keratoconus diagnostics with Scheimpflug photography. Ophthalmologe. 2014;111(10):920-26.

24. Grewal DS, Brar GS, Jain R, Grewal SPS. Comparison of Scheimpflugimaging and spectral domain anterior segment optical coherence tomography for detection of narrow anteriorchamber angles. Eye (Lond). 2011;25(5):603-11.

25. Gatzioufas Z, Seitz B. New aspects on biomechanics of the cornea in keratoconus. Ophthalmologe. 2013;110(9):81017.;.

26. Nielsen $\mathrm{K}$, Hjortdal J, Pihlman $\mathrm{M}$, et al. Update on the keratoconus genetics. Acta Ophthalmol. 2013;91(2):106-13.

27. Shapiro MP, France TD. The ocular features of Down's syndrome. Am J Ophthalmol. 1985;99(6):659-63.

28. Harneed A, Khakiq S, Ismail M, Anwar K, et al. A novel locus for Leber congenital amaurosis (LCA4) with anterior keratoconus mapping to chromosome 17p13. Invest Ophthalmol Vis Sci. 2000;41(3):629-33.

29. Robertson I. (1975) Keratoconus and the Ehlers-Danlos Syndrom: a new aspect of Keratoconus. Med Aust 1(18):571-3-

30. McMahon TT, et al. Longitudinal changes in corneal curvature in keratoconus. Cornea. 2006;25(3)296-305.

31. Shapiro MP, France TD. The ocular features of Down's syndrom. Am J Ophthalmol. 1985;99(6):659-63.

32. Harneed A, Khakiq S, Ismail M, Anwar K, et al. A novel locus for Leber congenital amaurosis (LCA4) with anterior keratoconus mapping to chromosome 17p13. Invest Ophthalmol Vis Sci. 2000;41(3):429-33.

33. Robertson I. Keratoconus and the Ehlers-Danlos Syndrom: a new aspect of keratoconus. Med J Aust. 1975;1(18):571-3.

34. Efron N. Contact lens induced changes in the anterior eye as observed in vivo with the confocal microscope. Science. Direct. Prog Retin Eye Res. 2007;26(4):398-436.

35. Ku JY, Niederer RL, Patel DV, et al. Laser scanning in vivo confocal analysis of keratocyte density in keratoconus. Ophthalmology 2008;115(5):845-50.

36. Kalinikos P, Efron N. On the etiology of keratocyte loss during contact lens wear. Invest Ophthalmol Vis Sci. 2004;45(9):3011-20.

37. Tsubota K, MashimaY, et al. Corneal epithel in keratoconus. Cornea. 1995;14(1):77-83.

38. Li Y, Tan O, Brass R, et al. Corneal epithelial thickness mapping by Fourier-domain optical coherence tomography in normal and keratoconic eyes. Ophthalmology. 2012;119(12):2425-33.

39. Wu S, Tao A, Jiang H, et al. Vertical and horizontal corneal epithelial profile using ultra-high resolution and long scan depth optical coherence tomography. PLoS One. 2014;9(5):e97962.

40. Silverman RH, Urs R, RoyChoudhury A, et al. Epithelial remodeling as basis for machine based identification of keratoconus. Invest Ophthalmol Vis Sci. 2014;55(3):1580-7.

41. Zhou W, Stojanovic A. Comparison of corneal epithelial and stromal thickness distributions between eyes with keratoconus and healthy eyes with corneal astigmatismus $<2.0$ D. PLoS One. 2014;9(1):e85994.
42. Pflugfelder S, Liu Z, Feuer W, Verm A. Corneal thickness indices discriminate between keratoconus and contact lens induced corneal thinning. Ophthalmology. 2002;109(12):2336-41.

43. Gasset AR, Haude WL, Garcia-Bengochea M. Hard contact lens wear as an environmental risk on keratoconus. Gaineswille, Florida. Am J Ophthalmol. 1978;85(3):339-41.

44. Levenson DS. Changes in corneal curvature with long term PMMA contact lens wear. CLAO J. 1983;9(2):123.

45. Macsai MS, Varley GA, Krachmer JH. Development of keratoconus after contact lens wear. Arch Ophthal mol.1990;108(4):534-8.

46. Tao X, Yu H, Zhang Y, et al. Role of corneal epithel in riboflavin/ultraviolet-A mediated corneal crosslinking treatment in rabbit eyes. Biomed Res Int. 2013;2013:624563.

47. Kaldawi RM, Wagner J, Ching S, et al. Evidence of apoptotic cell death in keratoconus. Cornea. 2002;21(2):2006-9.

48. Sykakis E, Carley F, Iroion L, et al. An in depth analysis of histopathological characteristics found in keratoconus. Pathology. 2012;44(3):234-39.

49. Patel DV, Ku JY, Johnson R, et al. Laser scanning in vivo confocal microscopy and quantitative aesthesiometry reveal decreased corneal innervation and sensation in keratoconus. Eye. 2008;23(3):586-92.

50. Mathew JH, Goosey JD, Bergmanson JP. Quantified histopathology of the keratoconic cornea. Optom Vis Sci. 2011;88(8):988-97.

51. Spoerl E, Huhle M, Seiler T. Induction of cross-links in corneal tissue. Exp Eye Res. 1998;66(1):97-103.

52. Spoerl E, Schreiber J, Hellmund K, et al. Untersuchungen zur Verfestigung der Hornhaut am Kaninchen [Studies on the stabiliszion of the cornea in rabbits]. Ophthalmologe. 2000;97(3):203-6.

53. Edmund C. Corneal elasticity and ocular rigidity in normal and keratoconic eyes. Acta Ophthalmol. 1988;66(2):134-40.

54. Wollensak G, Spoerl E, Seiler T. Riboflavin/ultraviolet-A induced collagen crosslinking for the treatment of keratoconus. Am J Ophthalmol. 2003;135(5):620-27.

55. Pollhammer M, Cursiefen C. Bacterial keratitis early after corneal cross- linkin with riboflavin and ultraviolet-A. J Cataract Refract Surg. 2009;35(3):588-89.

56. Peres-Santonja JJ, Artola A, Javaloy J, et al. Microbial keratitis after corneal collagen crosslinking. J Cataract Refract Surg. 2009;35(6):1138-40.

57. Sharma M, Maharana P, Singh G, et al. Pseudomonas keratitis after collagen crosslinking for keratoconus:case report and review of literature. J Cataract Refract Surg. 2010;36(3):517-20.

58. Garcia -Delpech S, Diaz-LIopis M, Udaondo P, et al. Fusarium keratitis 3 weeks after healed corneal crosslinking. J Refract Surg. 2010;26(2):994-5.

59. Rama P, Di Matteo F, Matuska F, et al. Acanthamoeba keratitis with perforation after corneal crosslinking and bandage contact lens use. J Cataract Refract Surg. 2009;35(4):788-91.

60. Angunawela RI, Arnalich_Montiel F, Allan BD. Peripheral sterile corneal infiltrates and melting after collagen crosslinking for keratoconus. J Cataract Refract Surg. 2009;35(3):606-7.

61. Arora R, Jain P, Gupta D, et al. Sterile keratitis after corneal collagen crosslinking in a child. Cont Lens Anterior Eye. 2012;35(5):233-5.

62. Kymionis GD, Portaliou DM, Diakonis VF, et al. Corneal collagen crosslinking with riboflavin and ultraviolet-A irradiation with thin corneas. Am J Ophthalmol. 2012;153(1):24-8. 
63. Gokhale NS. Corneal endothelial damage after collagen crosslinking treatment. Cornea. 2011;30(12):1495-98.

64. Bagga B, Pahuja S, Murthy S, et al. Endothelial failure after collagen crosslinking with riboflavin and UV-A: case report with literature review. Cornea. 2012;31(10):1197-200.

65. Sharma A, Nottage JM, Mirchia K, et al. Persistent corneal edema after collagen crosslinking for keratoconus. Am J Ophthalmol. 2012;154(6):922-6.

66. Alhayek A, Lu PR. Corneal collagen crosslinking in keratoconus and other eye disease. Int J Ophthalmol. 2015;8(2):407-18.
67. Vazirani J, Basu S. Keratoconus: current perspectives. Clinical Ophthalmol. 2013;7:2019-30.

68. Sorkin N, Varssano D. Corneal collagen crosslinking: a systematic review. Ophthalmologica. 2014;232(1):10-27.

69. Liu Z, Pflugfelder SC. The effect of long-term contact lens wear on corneal thickness, curvature, and surface regularity. Ophthalmology. 2000;107(1):105-11. 